\title{
Highlighting the role of Requirement Engineer
and User Experience Design in Product Development Life Cycle
}

\author{
Ambreen Nazir \\ Department of Software Engineering, University of Engineering and Technology, Taxila, Pakistan \\ Email: ambreen_nazir_ms@yahoo.com \\ Ayesha Raana \\ Department of Software Engineering, University of Engineering and Technology, Taxila, Pakistan \\ Email: ayesha.raana@yahoo.com \\ Nadeem Majeed \\ Department of Software Engineering, University of Engineering and Technology, Taxila, Pakistan \\ Email: nadeem.majeed@uettaxila.edu.pk
}

\begin{abstract}
Product Development Life Cycle (PDLC) has been evolving from last decade from side to side unremitting progression of finest practices, process models and advance life cycles. Already present development models like rational unified process, waterfall model and spiral model have facilitated the people, who are being practicing the new models, to incalculably make their products better on the competence and effectiveness. Nevertheless, there is a need to quantify the product quality with the factors that are away from any traditional criterion like maintainability, reliability and the rest. Accomplishing a product that is technically strong only contributes a little part in sensational product market situation, but to what extent the product fulfills the major needs of the users reveals the real success of the product. Requirement Engineering (RE) and User Experience Design (UED) together make available the comprehended user requirements to product development team for successful implementation of product and further help the development team to resolve any problem that takes place. The paper emphasizes on the role of RE and UED during PDLC and discusses the challenges that comes out after coalition of RE and UED in product development. In the closing section some points are listed for determining these challenges.
\end{abstract}

Index Terms - Challenges, (Product Development Life Cycle) PDLC, (Requirement Engineering) RE, (User Experience Design) UED.

\section{INTRODUCTION}

During PDLC, RE participation is considered very essential and critical. Performing requirements organization has turned out to be a critical intentional work in many organizations for successful development. As days go by organizations have to make contract with different stakeholders, deal with a huge number of requirements and make configurations among products and approaches for quickly advancing their goods. The integration of RE in PDLC is fully responsible for the recognition and performance of the product in the market place. For the flourishing product development, not only during the initial phases of PDLC but all through the way of PDLC, gathering user requirements from different stakeholders and dealing with users' practice is considered as foremost challenge of RE.

In PDLC, UED plays a major role which can't be ignored. If you can't make your users happy with the product you are developing, then your competitor will take advantage of this thing. Organizations should consider the users' interest and give special attention to the people for whom they are making all their goods.

Many organizations are still applying their customary practices during the PDLC without making users a part of the requirements in the initial stages. In past, several schemes and practices are proposed [1] [2] to incorporate user attention in PDLC but with some limitations. UED never finds an official place in the traditional product engineering, totally dissimilar to RE. That's why many organizations and product developing team are still unfamiliar with UED. As the stakeholder imaginations are enlarging with technology advancement, a simple SRS is not enough for handling all the user's needs. So it becomes necessary to concentrate on RE and UED during PDLC. The responsibility of RE and UED was initially given to product managers, engineers and product designers. But as time passes, a tremendous evolvement was noted in the fields of RE and UED that makes it complicated for a single product or architect or designer to handle all the duties and liabilities of RE and UED. Therefore, in this paper we have presented a multi disciplinary strategy where all the RE and UED experts will work mutually with the development team during the whole PDLC. 
This paper comprises of seven sections. In Section II, a critical review of the work done in PDLC during RE and UED is given. In Section III, we put light on the position of RE and UED disciplines in PDLC and on the responsibilities of RE and UED experts. In Section IV, a multidisciplinary approach is presented in which the RE and UED experts work together for their successful integration in PDLC. In Section V, we have highlighted the two major challenges that we can face due to collaborative work of RE and UED during PDLC. In Section VI, some preventive measures are taken into account for confronting the challenges of Section V. In Section VII, concluding remarks are given supporting future research directions.

\section{LITERATURE REVIEW}

A major mistake that is done during PDLC is the suspension of RE activities as the starting phase of process development starts and UED is left only for the tail end. This takes place only due to the mistaken belief and prospective of these disciplines. Setting up continuity of RE and UED activities all throughout PDLC not only makes easy for the product team to track and resolve changes but also keeps them alert about costing problems at each milestone.

For years a number of researchers have been working on linking requirements and usability. Neil Maiden [3] in his paper presented some problems in the projects that arouse due to treating the User and System requirements as the same thing are unpacked. Here are two such problems: First, when all requirements are treated as system requirements, stakeholders then only give you system requirements. Second, designers design the system by using user requirements but these requirements don't specify the system's properties. User and system requirements are of different types, user requirements express a property of the domain or business process that the introduction of a new system will bring about whereas implementation of system requirements in some business process, will lead to the achievement of at least one user requirement. Three strategies are discussed to differentiate between the two types of requirements: represent user and system requirements differently, manage the relationship between user and system requirements explicitly and designers use creativity techniques with analysts and stakeholders to invent system requirements to satisfy user requirements.

Anitha PC and Beena Prabhu [4] in their paper discussed some of the misconceptions and myths that exist in the industry regarding RE and UED, and how user needs typically slip through the cracks under the name of "non functional requirements". RE and UED focus during the overall PDLC is the real focus of this paper. An integrated framework for both RE and UED in the PLM process is proposed and they recommended that both RE and UED professionals should work in coordination throughout the PDLC to ensure that the relevant requirements are implemented, realized and validated in a systematic and measurable way as effective collaboration of RE and UED professionals throughout the PDLC is stressed to add value in building successful products. The integration of UED with PDLC would ensure the development of a product that has clear unique selling points and enable the adoption and sustainment of the product in the market.

Richard Berntsson Svensson, Tony Gorschek, Björn Regnell, Richard Torkar, Ali Shahrokni, Robert Feldt and Aybuke Aurum [5] discussed two perspectives of the prioritization of QR (Quality Requirements). First the product perspective, studied through the role of Product manager. Second the project perspective, studied through the role of Project leader. The main focus of this paper is to present the results of an empirical study that includes data collected through indepth interviews with different companies and to gain in-depth understanding of the nature of $Q R$ prioritization within market-driven embedded systems companies. Their results revealed that several companies prioritize the QR and FR (Functional Requirements) by using the same prioritization method, even though these two are very different. Also product management focus on FR to achieve a competitive advantage and may not using $\mathrm{QR}, \mathrm{QR}$ have given very low priority and only prioritize if time and resources are available once all FR have been implemented to the satisfactory level.

Christ of Ebert [6] in his paper showed that product duration, quality and schedule adherence is totally dependent upon the sound product management. Too often projects or product releases fail due to misunderstanding or misinterpreting customer needs and later on coping with associated changes. For successful product management the following factors are coined into guidelines: Business objectives and accountability; Mastering requirements; Managing risks and uncertainty; Leadership and team work. Business objectives and Requirements must be supervised (planned, prioritized, agreed, monitored) to assure focus and for this a product core team with product management, marketing (or sales) and engineering lead by the product manager is strongly recommended and there must be no lack of commitments among stakeholders.

According to Pascal Raabe [7], an iterative cycle where every single stage is measured with respect to the primarily well-known and well understood requirements of the stakeholders is termed as a user centric design process and this cycle is iterated till all the requirements are fulfilled. He clearly mentioned that UED should be applied to all the design areas, such as web design, architecture design, interface design editorial design, product design, service design, way finding, urban design, for granting high quality user experience. He proposed a variety of techniques and methods to formulate enhancement in design by considering user needs. These techniques and methods are Sticky Notes, Project Space, Good Practice Guidelines, Aesthetics, Prototyping, User Requirements, Persona and Scenarios, Progressive Disclosure, Usability Testing and Rapid Visualization. 
Mike Guiltier [8] in his research expressed top four practices of UED that helps the product developers to form high experience from the user that is helpful, useable and demanding. His first practice was "Become your Users"; in this he told how to interact with users, how to listen in to their needs; how to monitor your users habits in a natural environment and how to give them special attention to enhance their confidence. His second practice "Design First" was about finding how one can complete all the user needs in a particular business domain; how to produce design talent that starts with a low-fidelity prototype, this design should be flexible and it should have the ability of managing change by residing within certain limits. Third practice "Trust No One-Test" was to make ensure that end user is happy and satisfied; this can be done by testing the product again and again and by taking different expert reviews. Fourth practice "Inject UED into your PDLC" was about how to inform every member of the team about the importance of UED and what is it; how to pick out a leader who will promote and compel UED; how to renovate your application so that it can accept UED.

Paul Sherman and Sherman UX [9] provided the research for the people who are highly concerned with having a deep know how of UED. They gave a brief summary of UED design and techniques and how UED can be integrated into the processes during PDLC. They also described some complexities normally come across when any product organization going to build UED team.

\section{PRODUCT DEVELOPMENT LIFE CYCLE (PDLC)}

PDLC [10] deals with each and every one activity that goes into developing a product, from the commencement until the dumping process. Manufacturing of the product truly verifies what measures should be taken afterward in all phases of product development. For a product to survive in the operating environment is a real challenge from practical viewpoint as it has to keep evolving it features in order to retain its status in market. Five major periods of PDLC are:

\section{A. Product Development Phase}

This phase starts, whenever any new idea is discovered and implemented by the organization. This includes transforming a variety of information and fitting it into an innovative product. A product is processed several times before representing it to the stakeholders and for this a lot of money and time is needed. The product that continues to exist in the test market is then launched into real marketplace where the introduction phase of the product begins.

\section{B. Introduction Phase}

This phase of a product takes account of launching the product with its entire requirements in a way that product will gain the utmost brunt at the sale flash. Introduction phase is the critical phase for the product as the chances of drowning money at this period are much more than the maturity phase of the product. The flourishing product introduction phase is totally dependent on the actions that the company takes before introducing the product to the market.

\section{Growth Phase}

This phase is actually the real satisfaction of considering the product prospering at the marketplace. And the perfect timings of focusing on escalating market share are when your product gains much success. A high-quality act to promote and advertise your product at this stage is the utilization of promotional contractors from the outdoor. All through the growth phase excellent exposure in the market areas is a valuable goal.

\section{Maturity Phase}

This phase appears when market is flooded with different essential products, and all the opponents are signified in condition of a substitute product. It's the time when someone else's business expenditure is the real cause of the market share growth. The stage is period of getting utmost profit from the product. New brands are also launched during this phase even they are vying with exiting products of the company. Frequently models are changed to widen the life of the product.

\section{E. Decline Phase}

The choice of diminishing a product appears to be an intricate task and a bundle of concerns should be resolved before withdrawing the product away from the market. Problems for instance maintenance, availability of extra parts, tune-up competitions response in satisfying the market hole are several issues that amplifies the complications of the decision process of withdrawing a product from the market. Occasionally because of big product success in the maturity phase declination of the product becomes difficult.

The phases of PDLC are shown in Fig. 1.

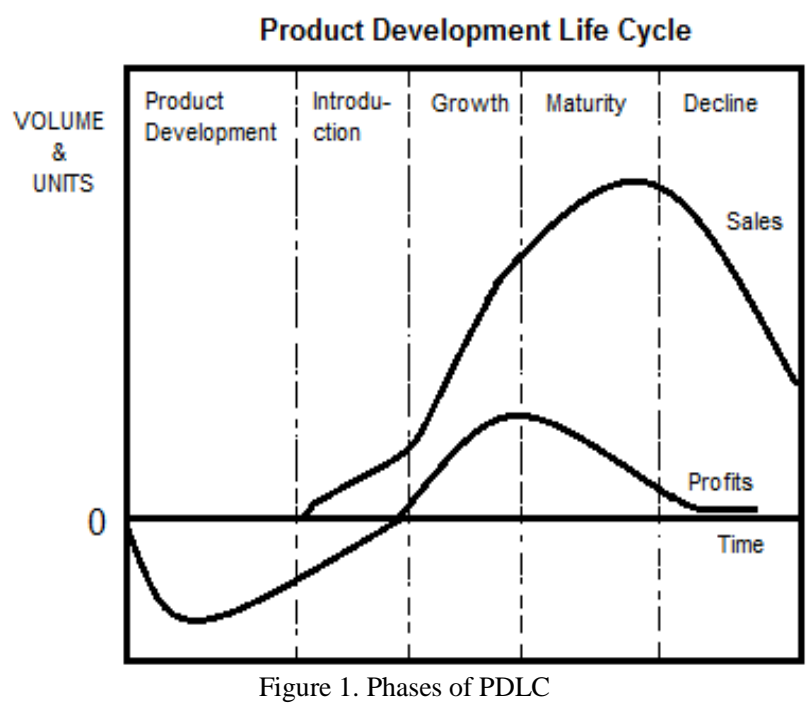

Requirements formation and organization is vital for successful development of many products and for decades this thing has been carried out without the contribution of devoted RE and UED experts. In present 
days, market demands are varying with dynamism, technology is becoming advance day by day and user trends are changing. So, to cope with all the advancement it becomes imperative to have capable and keen RE and UED experts during the product development that carries out requirements engineering and user experience activities. RE and UED brings forward many areas that may be uncertain, in this section we have summarized the responsibilities and benefits of these areas.

\section{F. Role of RE in PDLC}

RE expert helps in supplying a connection among people (clients, intermediate persons, end users, management team, sales person, marketing team, authoritarian, challengers and developers) who are openly or ultimately concerned about the product which is under development. For all the tasks and activities that are performed during $\mathrm{RE}$ lifecycle, $\mathrm{RE}$ expert is accountable. A good RE expert should acquire well sturdy communication and conciliation skills as well as he should have the ability to connect with other personals together with technological and field awareness for dealing with all activities effectively and in a quick manner. The duty of any RE expert starts even before the requirement phase in conception phase which is called 'requirement that is needed previous to requirements', in this product supervision team of the organization does all the scheduling and identifies their aim for production and business.

Basically, RE expert engages himself in recreating to achieve the activity of eliciting the requirements that helps in identifying the stakeholders' desires by means of different methods like suggestion meeting, exploration events, data representing techniques, rough sketching and so on. He smoothly examines and purifies all the needs of concerning people into proper product specifications for making direct communication with them. He measures the quality and validates every single requirement by using some standard and he also facilitates in managing requirement activities by setting up change control board (CCB), setting up end to end requirements tracing mechanism and verification and validation of the realized requirements.

When RE analysis is done by any inexpert person, it might end in inaccurate stakeholders needs for determining the minimum marketable features of the product; gathered information may be vague to get awareness about the product wants; there might be chances of additional pointless redundant requirements into the product extent.

\section{G. Role of UED in PDLC}

User Experience exemplifies the product along with experiencing the user interface. A real significant product always provides some benefit to the user so he/she would persistently connect with it. We can imagine the significance of UED [9] in any PDLC that it plays vital part in assembling all the figures and information that is directly connected to the consumer and market-place. It interprets and clarifies all the gathered data into convincing answer for all the all user and ecological unit. UED expert has the liability of manipulating the complete understanding of the user experience with the product that how he will accept, acclimatize and perceive about the product. In order to meet the stakeholder needs, ambitions and limitations and also to assure all the technological limitations, business objectives and company goals, practically and bodily, all the communication among the user and the product must be designed.

Throughout PDLC, UED plays a major role in targeting the market segments by using different methods like segmentation models, profiles and personals, in getting a clear view about the market place by studying different landscapes, in perceiving the user and its interaction with environment with the help of ethnographic interviews and society investigation, in understanding how the user will accept the product by making comparable and contributory designs. UED expert always concentrates on creating the products that are easy to use, valuable and proficient. He adopts an iterative approach for the distillation of the design using prototypes by performing decisive testing and in time validation.

UED in PDLC makes it certain that the product would be having obvious exclusive selling position and also it would make possible the acceptance and continuity of the product in the market-place.

\section{INTEGRATION OF RE AND UED IN PDLC}

PDLC covers all the phases for supervising the total improvement of a product from its commencement, during design and apprehension, to support and end stage. PDLC acts as an integration media framework among stakeholders, practices and business features. To be on the top level of organization's business policy, many companies have changed and unmitigated their previous product development processes. In PDLC, RE takes an official recognized place and all RE development and management works in iterative and parallel manner. But, UED requirements which are of extreme importance and are extensive in natural world are frequently mistreated. Generally, a large amount of organizations do not accept the integrated UED seamlessly.

In this part, we offer a framework after integrating both RE and UED in the PDLC process and we suggest that throughout the PDLC process both RE and UED experts should work collaboratively to make certain that the gathered necessary requirements are applied, authenticated and validated in efficient and quantifiable way. A number of harmonization spots are acknowledged where RE and UED experts should cooperate with each other and the recommended framework is shown in Fig. 2.

In PDLC, conception phase is the initial spot where both experts collaborate with each other. As the RE expert done about extracting requirement about the 
business objectives, product ambition and stakeholder investigation result is shared with the UED expert so that he/she got the clear imagination about the product perception that will help him/her in studying the users to determine how much this product will be beneficial for them and to get an apparent imagination that how the users will take the product. The result gained by the conception phase of UED is participated as an input key for the analysis stage of RE where RE experts perform the business and technological practicability test to baseline the approved and certified requirements with the assistance of other stakeholders like area specialist, planner and product manager. This is the point where both the RE and UED association is done in order to pay attention to business policies and how much these policies satisfy the user wants.

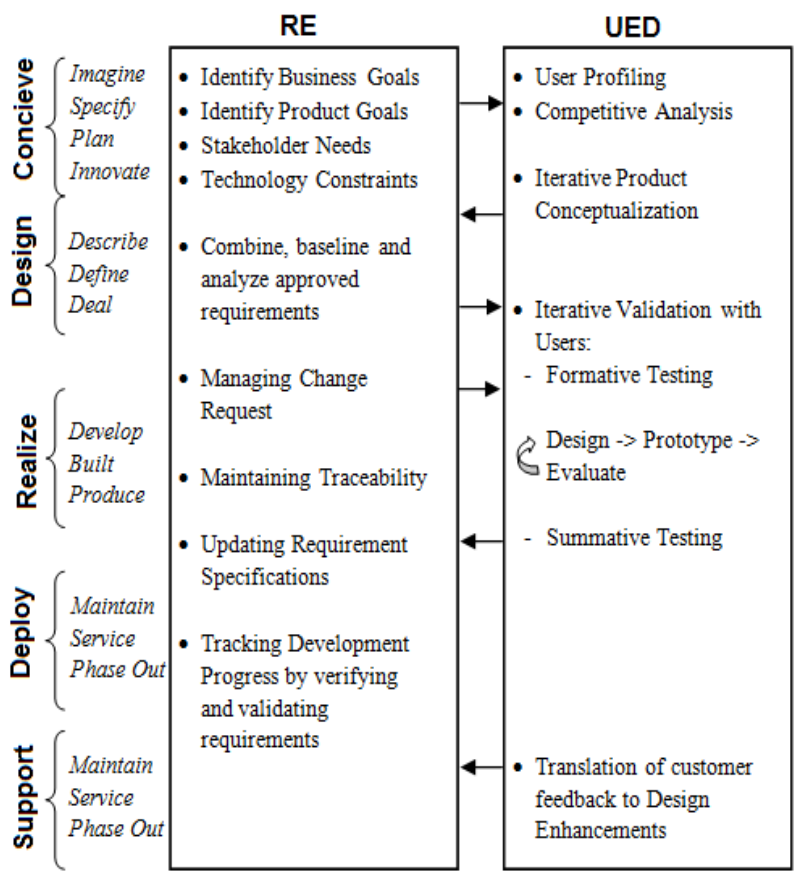

Figure 2. Integrated Framework of RE and UED in PDLC

When all the baseline requirements are prepared, the RE expert has to align up with the UED expert for providing explanations and details to the product development team so that the realization of the product can be started without any delay. Throughout the realization phase from side to side with the deployment phase of PDLC process, both RE and UED experts have to interact with each other in a continuous manner for handling and managing the demands of changing requirements which are related to user experience and also for authenticating and validating the realized requirements by executing formative and summative testing continuously and iteratively. The RE and UED experts work with each other during the support phase for collecting the user's response and opinion, then communicating them again to the product development team. During the support phase it becomes probable to observe the product functioning in the real surroundings that makes possible to fix problems and plan for the next version. Background investigation are librated from presumptions and limitations forced by usability testing and assist user investigators to observe how users utilize a product and what is their purpose behind using a product. The feedback we get from the users in the support phase is very supportive and becomes a basis of information that afterward helps in making further advancements in the user interface as well as user content helps in defining additional features for the next releases. For bringing innovation in the PDLC process, we determine that RE and UED collaboration plays a significant part.

\section{CONFRONTING CHALLENGES DUE TO COLLABORATION OF RE AND UED}

Due to group effort of RE and UED experts in PDLC, the product development team have to confront many challenges because of deficiency of knowledge they need for developing the product. From years even though, many researchers are delivering words about fulfilling the gap between RE and usability, by bringing the usability into the initial phases of product development. These challenges keep on manipulating the best practices that the organizations follow for developing the product. The two major challenges that we face are listed below.

1. Firstly, user requirements should be stated in such layout that requirement engineers can effortlessly add these requirements into their pomposidad requirements credentials.

2. The division of user requirements into definite design elements that helps the development people in implementing these requirements.

Further we will discuss, what measures we should take to resolve these challenges that we confront by mutual cooperation of RE and UED in PDLC.

\section{HOW TO RESOLVE THESE CHALLENGES?}

RE and UED experts excess the SRS (System Requirement Specification) for jumping on text step of PDLC, where they work together to come up with enhanced and improved requirements piece of art. RE experts give all their attention to ERS (Engineering Requirement Specification) while UERS (User Experience Requirement Specifications) is the center of attention for UED experts continually with the users. We listed down the preventive actions that should be taken before finalizing the requirements to overcome the above mentioned challenges.

- RE and UED experts should bring to light the real distinction between the user requirements and the system requirements. Because amalgamation of user and system requirements can direct too many confusions. As product developers only give impression of user requirements during the designing but system possessions can't be identified by the user requirements. On the other side if product developers use extravagance of system requirements then this 
might not go ahead with accomplishing actual user requirements which are not documented.

- RE and UED should trace each and every user and system requirement. It will facilitate by supplying two-way traceability among diverse connected requirements. It will permit $\mathrm{RE}$ experts to follow all the modifications that will be made to these requirements plus to discover the foundation of every requirement.

- Requirements should be prioritized by RE and UED experts by using some effective techniques. As it is most demanding action in PDLC and it is very essential for a product to be successful to discover the correct stability between challenging requirements.

- Requirements should only be delineated after receiving contribution from some stakeholders, so that consequential problem definition becomes clearly comprehensive for developing the product.

- Concluding requirements should be authenticated in the presence of stakeholders after assembling all the requirements from them to avoid any complexities during PDLC.

- All the conflicts and incompatibilities of requirements should be resolved by RE and UED expert eventually.

- Write up of all requirements should be to a level that can be easily understood by all stakeholders so that everyone does not interpret the requirement statement from its own view point. Requirements notations and procedures must preserve slight stability in the middle of producing descriptions that create appropriate technical documents that helps out downstream developers.

- Elaboration of all the requirements should be in breadth first manner to restrain form high possibility of re-work once the complete explanation is explicitly done in breadth first manner.

- SRS should be finely structured and base lined to maintain clearness.

- Whenever requirements are modified then change management process should be fully controlled.

- Do not throw away all your exertion in providing complete description of invaluable requirements in SRS.

\section{CONCLUSION AND FUTURE WORK}

During product development processes, RE and UED becomes obligatory. The basic intention of this paper is to grant awareness about their roles and to increase their incorporation in PDLC as effective teamwork of RE and UED experts contribute great part in product formation and maintenance and also confirms building successful products. Further, we proposed an incorporated framework for both RE and UED in PDLC and make certain that their collaboration will assist in implementing requirements that will be authenticated in an organized and quantitative way. We also mentioned the challenges that can be resulted by mutual integration of RE and UED and listed of some point that will help in resolving these challenges.

Our future work will be the examination of these mentioned challenges by means of case studies and under development ventures.

\section{REFERENCES}

[1] Luiz Marcio Cysneiros and Andre Kushniruk , IEEE, "Bringing Usability to the Early Stages of Software Development", http://citeseerx.ist.psu.edu/viewdoc/download?doi= 10.1.1.106.7771\&rep=rep1\&type=pdf, 2003.

[2] David Callele, Eric Neufeld, Kevin Schneider, IEEE, "An Introduction To Experience Requirements", http://ieeexplore.ieee.org/xpl/login.jsp?tp=\&arnumb er=5636572\&url=http $\% 3 \mathrm{~A} \% 2 \mathrm{~F} \% 2$ Fieeexplore.ieee. org\%2Fxpls\%2Fabs_all.jsp\%3Farnumber\%3D5636 572,2010

[3] Neil Maiden, IEEE, "User Requirements and System Requirements", http://ieeexplore.ieee.org/stamp/stamp.jsp?tp=\&arn umber $=4455639$, April 2008.

[4] Anitha PC, Beena Prabhu, IEEE, "Integrating Requirements Engineering and User Experience Design in Product Life Cycle Management", http://ieeexplore.ieee.org/stamp/stamp.jsp?tp=\&arn umber=6226784, 2012.

[5] Richard Berntsson Svensson, Tony Gorschek, Björn Regnell, Richard Torkar, Ali Shahrokni, Robert Feldt, Aybuke Aurum, IEEE, "Prioritization of Quality Requirements: State of Practice in Eleven Companies",http://ieeexplore.ieee.org/stamp/stamp. jsp?tp=\&arnumber=6051652, 2011.

[6] Christof Ebert," The impacts of software product management",http://www.compaid.com/caiinternet/ ezine/ebert-impacts.pdf, October 27, 2006.

[7] Pascal Raabe, "User Centered Design", http://paznow.s3.amazonaws.com/User-CentredDesign.pdf, 2010.

[8] Mike Gualtieri, Best Practices In User Experience (UX) Design, http://wwwimages.adobe.com/www.adobe.com/con tent/dam/Adobe/en/industryinsights/solutions/pdfs/ Forrester_Best_Prac_In_User_Exp.pdf, September 4, 2009.

[9] Paul Sherman, Sherman UX, "The User Experience Team Kit: How to Hire a UX Team and Incorporate User-Centered Design Methods into Your Software Development Lifecycle Process", http://www.ixda.org/sites/default/files/UX_Kit_Aug 09.pdf, Version 1.1, February 212010.

[10] Product Life Cycle Management, "What is PLM", http://www.product-lifecycle-management.info/, 2011. 
Ms. Ambreen Nazir is a MS Scholar as well as Research Associate in the Department of Software Engineering at University of Engineering and Technology Taxila, Pakistan. She graduated from University of Engineering and Technology Taxila in Software Engineering in July 2012. Her areas of interest are Windows Application Development, Web Development, Databases, Software Quality Assurance, Software Project Management, and Software Requirement Engineering. She is trying to bring innovations in above mentioned fields.

Ms. Ayesha Raana is a MS Scholar as well as Research Associate in the Department of Software Engineering at University of Engineering and Technology Taxila, Pakistan. She graduated from University of Engineering and Technology Taxila in Software Engineering in July 2012. Her areas of interest are Software Requirement
Engineering, Software Quality Assurance and Operating System.

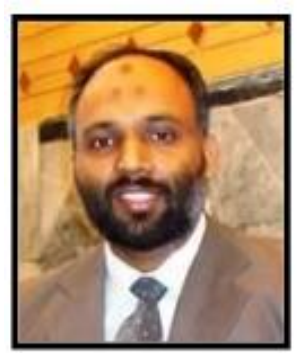

Mr. Muhammad Nadeem Majeed is $\mathrm{PhD}$ Scholar in Department of Computer Engineering at University of Engineering \& Technology Taxila. He holds a MS degree in Computer Engg. from Center for Advance Engineering, University of Engineering \& Technology Taxila and has 11 years teaching experience. His areas of interest are Computer Communication and Networks, Wireless Networks, Ad-hoc Networks, Software Requirement Engineering, Software Project Management and Advance Operating System. Nadeem has been striving to bring innovations in the said fields through his research.

How to cite this paper: Ambreen Nazir, Ayesha Raana, Nadeem Majeed,"Highlighting the role of Requirement Engineering and User Experience Design in Product Development Life Cycle", IJMECS, vol.6, no.1, pp. 34-40, 2014.DOI: 10.5815/ijmecs.2014.01.04 